\title{
Hydrogen transfer reduction of nitriles in DBU based ionic liquids
}

\author{
Xinzhi Chen, Shaodong Zhou, and Chao Qian* \\ Department of chemical and biological engineering, Zhejiang University. Zheda Road, \#38, \\ Hangzhou, 310027, China \\ E-mail: chemtec@163.com
}

DOI: $\underline{\text { http://dx.doi.org/10.3998/ark.5550190.0013.812 }}$

\begin{abstract}
Raney nickel catalyzed transfer hydrogenation of nitrile was proposed, with formats as hydrogen donor. Three ionic liquids, including $\left[\mathrm{DBUH}^{+}\right]\left[\mathrm{C}_{2} \mathrm{H}_{5} \mathrm{COO}^{-}\right],\left[\mathrm{DBUH}^{+}\right]\left[\mathrm{C}_{3} \mathrm{H}_{7} \mathrm{COO}^{-}\right]$, and $\left[\mathrm{DBUH}^{+}\right]\left[\mathrm{C}_{4} \mathrm{H}_{9} \mathrm{COO}^{-}\right]$, were prepared as reaction medium, and $\left[\mathrm{DBUH}^{+}\right]\left[\mathrm{C}_{4} \mathrm{H}_{9} \mathrm{COO}^{-}\right]$showed the best performance. Various nitriles were reduced with potassium formate as hydrogen donor, and considerable to excellent yields of amines were given. The ionic liquid can be used for 6 times at least, and the reaction process is clean.
\end{abstract}

Keywords: Catalytic transfer hydrogenation, reduction of nitriles, DBU based ionic liquid

\section{Introduction}

Catalytic transfer hydrogenation plays an important role in organic synthesis. Reduction of nitrile yields amine. There are many reductants that are used in the reduction of nitrile, such as hydrogen, ${ }^{1}$ sodium tetrahydroborate,${ }^{2}$ Aluminum, ${ }^{3}$ lithium aluminium hydride, ${ }^{4}$ et al. However, there are only a few reports on transfer hydrogenation system that is available for the reduction of nitriles. R.C. Mebane, et al. have reported Raney nickel catalyzed transfer hydrogenation of aliphatic nitrile using 2-propanol as hydrogen donor, and good yields of corresponding amines were given ${ }^{5}$. But when we perform their method in the reduction of aromatic nitrile, little corresponding amine was obtained. E. Mizushima et al. reported similar result, they found low yield of benzylamine $(6 \%)$ in $\mathrm{RuH}_{2}\left(\mathrm{PPh}_{3}\right)_{4}$ catalyzed reduction of benzonitrile with 2-propanol as hydrogen donor ${ }^{6}$. The transfer hydrogenation system, $\mathrm{Pd} / \mathrm{HCOOH}$, has been also reported to be used in the reduction of nitrile, ${ }^{7}$ but it showed bad performance. Raney nickel catalyzed transfer hydrogenation of nitrile with hydrazine hydrate as hydrogen donor has been reported by both W.W. Zajac, et al. ${ }^{8}$ and Q.D. You, et $a l,{ }^{9}$ and moderate yield of corresponding amine was 
obtained. Although hydrazine hydrate is an effective hydrogen donor, it is limited by its toxicity and explosibility. Recently, a new hydrogen donor, hydrazinium monoformate, was proposed and used in the reduction of nitrile. It is reported to be so effective that nitriles can be reduced in a short time with good to excellent yields of corresponding amines ${ }^{10}$. However, the active hydrogen comes from hydrazinium, and the formate anion is wasted.

In our recent study, we found that formate can be an effective hydrogen donor in Raney nickel catalyzed reduction of nitriles if the hydrolysis and alcoholysis can be inhibited. Hydrolysis and alcoholysis of nitriles is inevitable when water or alcohol is used as solvent, so choosing an appropriate reaction medium is vital.

In recent years, ionic liquids (ILs) are attracting more and more attention as green and efficient reaction media for catalytic transfer hydrogenation ${ }^{11}$. As the solubility of salts is higher in IL than in organic solvents, we chose an IL as reaction medium. Recently, it was found by our group that 1,8-diazabicyclo[5.4.0]undec-7-ene (DBU) based ILs were superior as catalyst in some organic reactions such as aza-Michael additions ${ }^{12}$ and Knoevenagel condensation, ${ }^{13}$ and we found it was also a good solvent. DBU based IL is attracting growing attention as it can be prepared by simple neutralization of DBU and acid. In this work, we prepared several DBU based ILs as solvent in Raney nickel catalyzed transfer hydrogenation of nitrile.

\section{Results and Discussion}

DBU based ILs were prepared through simple neutralization of DBU and various acids. Three organic acid, including propionic acids, butyric acid and pentanoic acid, were used to prepare corresponding ILs, which were $\left[\mathrm{DBUH}^{+}\right]\left[\mathrm{C}_{2} \mathrm{H}_{5} \mathrm{COO}^{-}\right], \quad\left[\mathrm{DBUH}^{+}\right]\left[\mathrm{C}_{3} \mathrm{H}_{7} \mathrm{COO}^{-}\right]$, and $\left[\mathrm{DBUH}^{+}\right]\left[\mathrm{C}_{4} \mathrm{H}_{9} \mathrm{COO}^{-}\right]$. The reduction of benzonitrile with sodium formate as hydrogen donor was selected as model to compare the performance of different reaction media, including water, ethanol, toluene and the ILs mentioned above. As water is an indispensable component in formate participated transfer hydrogenation, the optimum amount of water was also explored. It is clear in Table 1 that the optimum molar ratio $n(\mathrm{HCOONa}): n\left(\mathrm{H}_{2} \mathrm{O}\right)$ is 2 . The results show that transfer hydrogenations performed in ILs gave better yields than those performed in other solvents. Reactions carried out in water and ethanol failed to give considerable yields, and those can be attributed to hydrolysis and alcoholysis of benzonitrile. The reaction carried out in toluene took 14 hours to give $80 \%$ yield of benzylamine, which could demonstrate the promoting effect of the DBU based ILs. The comparison also showed the performance order of the three ILs, that $\left[\mathrm{DBUH}^{+}\right]\left[\mathrm{C}_{2} \mathrm{H}_{5} \mathrm{COO}^{-}\right]<\left[\mathrm{DBUH}^{+}\right]\left[\mathrm{C}_{3} \mathrm{H}_{7} \mathrm{COO}^{-}\right]<\left[\mathrm{DBUH}^{+}\right]\left[\mathrm{C}_{4} \mathrm{H}_{9} \mathrm{COO}^{-}\right]$. As the acidity order of the anions is $\mathrm{C}_{4} \mathrm{H}_{9} \mathrm{COOH}<\mathrm{C}_{3} \mathrm{H}_{7} \mathrm{COOH}<\mathrm{C}_{2} \mathrm{H}_{5} \mathrm{COOH}$, the performance of DBU based IL as solvent in transfer hydrogenation of nitrile depends on how weak the acidity of anion is. Thus it can be judged that the promoting effect of DBU based IL probably resulted from its basicity. Furthermore, we found that the solubility of salt in DBU based IL is low at room temperature, 
while it increases remarkably as the temperature rises. So it is convenient for separation after the reaction. $\left[\mathrm{DBUH}^{+}\right]\left[\mathrm{C}_{4} \mathrm{H}_{9} \mathrm{COO}^{-}\right]$was chosen as reaction medium for further investigation.

Table 1. Catalytic transfer hydrogenation of benzonitrile in various reaction media

\begin{tabular}{llc}
\hline Entry & Reaction medium & Yield $^{\mathrm{d}}(\%)$ \\
\hline 1 & Toluene & $12^{\mathrm{b}}$ \\
2 & Toluene & $55^{\mathrm{b}}$ \\
3 & Toluene & $53^{\mathrm{b}}$ \\
4 & Toluene & $32^{\mathrm{b}}$ \\
5 & Water & $15^{\mathrm{c}}$ \\
6 & Ethanol & $33^{\mathrm{c}}$ \\
7 & {$\left[\mathrm{DBUH}^{+}\right]\left[\mathrm{C}_{2} \mathrm{H}_{5} \mathrm{COO}^{-}\right]$} & $65^{\mathrm{c}}$ \\
8 & {$\left[\mathrm{DBUH}^{+}\right]\left[\mathrm{C}_{3} \mathrm{H}_{7} \mathrm{COO}^{-}\right]$} & $71^{\mathrm{c}}$ \\
9 & {$\left[\mathrm{DBUH}^{+}\right]\left[\mathrm{C}_{4} \mathrm{H}_{9} \mathrm{COO}^{-}\right]$} & $85^{\mathrm{c}}$ \\
\hline
\end{tabular}

${ }^{\text {a}}$ Reaction conditions: benzonitrile (50 mmol), HCOONa (200mmol), catalyst (Raney nickel, 0.5 $\mathrm{g}$ ), temperature $\left(70^{\circ} \mathrm{C}\right)$, time $(8 \mathrm{~h}){ }^{\mathrm{b}}$ The hydrogen donor for Entry 1 4 are anhydrous HCOONa, and the molar ratios of $\mathrm{n}(\mathrm{HCOONa}): \mathrm{n}\left(\mathrm{H}_{2} \mathrm{O}\right)$ are $1,2,3$ and 4 respectively. ${ }^{c}$ The hydrogen donor for Entry $5 \sim 8$ is $\mathrm{HCOONa} \cdot 2 \mathrm{H}_{2} \mathrm{O} .{ }^{\mathrm{d}} \mathrm{GC}$ yield.

After the reaction medium was selected, we compared the performance of different formates, the results are shown in Table 2. It can be seen that sodium formate, tetraethylammonium formate and potassium formate showed comparative performance, while reaction using triethylammonium formate as hydrogen donor did not give any benzylamine. The probable reason was that triethylammonium formate could decompose to triethylamine and formic acid when heated, and benzonitrile reacts with formic acid to give benzaldehyde under catalyze of Raney nickel ${ }^{14}$. Potassium formate was selected as hydrogen donor for further investigation.

Table 2. Catalytic transfer hydrogenation of benzonitrile in $\left[\mathrm{DBUH}^{+}\right]\left[\mathrm{C}_{4} \mathrm{H}_{9} \mathrm{COO}^{-}\right]$with different formates $^{\mathrm{a}}$

\begin{tabular}{llc}
\hline Entry & Formate & Yield $^{\mathrm{b}}(\%)$ \\
\hline 1 & HCOONa & 85 \\
2 & HCOOK & 89 \\
3 & HCOOH $\left(\mathrm{Et}_{3} \mathrm{~N}\right)$ & 0 \\
4 & $\mathrm{HCOO}\left(\mathrm{Et}_{4} \mathrm{~N}\right)$ & 86 \\
\hline
\end{tabular}

aReaction conditions: IL (50 mL), benzonitrile (50 mmol), hydrogen donor (200 mmol), water (400mmol), catalyst (Raney nickel, $0.5 \mathrm{~g})$, temperature $\left(70{ }^{\circ} \mathrm{C}\right)$, time $(8 \mathrm{~h}) .{ }^{\mathrm{b}} \mathrm{GC}$ yield. 
We also tried two commonly used reduction catalyst, $\mathrm{Pd} / \mathrm{C}$ and $\mathrm{Pt} / \mathrm{C}$, in the transfer hydrogenation of benzonitrile to compare with Raney nickel. The results are shown in Table 3. Whatever the solvent was, almost no reaction occurred when $\mathrm{Pd} / \mathrm{C}$ was used as catalyst. And the only product in $\mathrm{Pt} / \mathrm{C}$ catalyzed reaction was benzamide. Both $\mathrm{Pt} / \mathrm{C}$ and $\mathrm{Pd} / \mathrm{C}$ were thus proved to be inactive in the reduction of nitrile, while $\mathrm{Pt} / \mathrm{C}$ can be a good catalyst for the hydrolysis of nitrile.

Table 3. Reaction between benzonitrile and $\mathrm{HCOOK}$ catalyzed by $\mathrm{Pd} / \mathrm{C}$ and $\mathrm{Pt} / \mathrm{C}^{\mathrm{a}}$

\begin{tabular}{llll}
\hline Entry & Reaction medium & $\begin{array}{l}\text { Product }(\text { yield } \\
\text { Pd/C catalyzed } \\
\text { reaction }\end{array}$ & $\begin{array}{l}\text { Product }\left(\text { yield }^{\mathrm{b}}\right) \text { in } \mathrm{Pt} / \mathrm{C} \\
\text { catalyzed reaction }\end{array}$ \\
\hline 1 & Water & Benzamide $(2 \%)$ & Benzamide $(>99 \%)$ \\
2 & Toluene & Benzamide $(<1 \%)$ & Benzamide $(30 \%)$ \\
3 & {$\left[\mathrm{DBUH}^{+}\right]\left[\mathrm{C}_{4} \mathrm{H}_{9} \mathrm{COO}^{-}\right]$} & Benzamide $(<1 \%)$ & Benzamide $(40 \%)$ \\
\hline
\end{tabular}

${ }^{\text {a}}$ Reaction conditions: Rolvent (50 mL), benzonitrile (50 mmol), HCOOK(200 mmol), water(400 mmol), catalyst $(0.5 \mathrm{~g})$, temperature $\left(70^{\circ} \mathrm{C}\right)$, time $(8 \mathrm{~h}) .{ }^{\mathrm{b}} \mathrm{GC}$ yield.

With the optimized reaction condition obtained, we expanded our study to other nitriles, and the results are summarized in Table 4. Generally various nitriles were reduced with good yields of corresponding amines. The reduction product of 4-chlorobenzonitrile was benzylamine, and the nitro group in 4-nitrobenzonitrile was reduced to amino. So it can be concluded that this transfer hydrogenation system is also available in dehalogenation and reduction of nitro. It should be noted that the reduction of aliphatic nitriles took much shorter time than that of aromatic nitriles (Table 4, Entry 1 and 2). This can be attributed to the conjugation of $\mathrm{C} \equiv \mathrm{N}$ bond and benzene ring, which reduced the electron density of the cyano group so that the hydrogenation is unfavorable. In order to exclude the effect of steric hindrance to the reaction, transfer hydrogenation of phenylacetonitrile was performed. It is clear in Table 4 that phenylacetonitrile was reduced in short time with good yield of phenylethylamine (Table 4, Entry 8), in agreement with our assumption. It took longer time for the transfer hydrogenation of 2-methylbenzonitrile, probable due to the steric hindrance of the ortho methyl group.

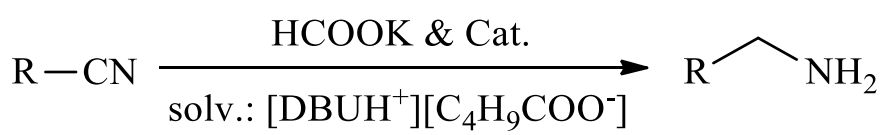

Subsequently, the reusability of $\left[\mathrm{DBUH}^{+}\right]\left[\mathrm{C}_{4} \mathrm{H}_{9} \mathrm{COO}^{-}\right]$was investigated, and transfer hydrogenation of benzonitrile was selected as the model reaction. After the reaction in every cycle, amine and unconverted nitrile was extracted with ethyl acetate, and the salts deposited at 
room temperature. After filtration, the IL was washed by ethyl ether and dried at $70{ }^{\circ} \mathrm{C}$ under vacuum for 2 hours. Then it was ready to reuse in next cycle. The results are shown in Table 5. It can be seen that the performance of $\left[\mathrm{DBUH}^{+}\right]\left[\mathrm{C}_{4} \mathrm{H}_{9} \mathrm{COO}^{-}\right]$maintained even when it was recycled for 6 times.

Table 4. Catalytic transfer hydrogenation of different nitriles ${ }^{\mathrm{a}}$

\begin{tabular}{llllll}
\hline Entry & $\begin{array}{l}\mathrm{R} \\
\text { (except for Entry }\end{array}$ & Time (h) & Product & $\begin{array}{l}\text { GC } \\
(\%)\end{array}$ & $\begin{array}{l}\text { yield } \\
\text { Isolated yield } \\
(\%)\end{array}$ \\
\hline 1 & Ethyl & 3 & $\mathbf{1 a}$ & 90 & 85 \\
2 & n-Propyl & 3 & $\mathbf{1 b}$ & 88 & 82 \\
3 & Phenyl & 8 & $\mathbf{1 c}$ & 89 & 86 \\
4 & 4-Tolyl & 8 & $\mathbf{1 d}$ & 91 & 87 \\
5 & 2-Tolyl & 14 & $\mathbf{1 e}$ & 70 & 65 \\
6 & 4-Chlorophenyl & 8 & $\mathbf{1 c}$ & 78 & $71^{\mathrm{d}}$ \\
7 & 4-Methoxyphenyl & 8 & $\mathbf{1 g}$ & 81 & 74 \\
8 & Benzyl & 4 & $\mathbf{1 h}$ & 87 & 84 \\
9 & 4-Nitrophenyl & 8 & $\mathbf{1 i}$ & 85 & 83 \\
10 & 1H-indol-2-yl & 8 & $\mathbf{1 j}$ & 78 & 75 \\
11 & Isophthalonitrile $^{\mathrm{c}}$ & 10 & $\mathbf{1 k}$ & - & 70 \\
\hline
\end{tabular}

aReaction conditions: IL (50 mL), nitriles (50 mmol), HCOOK (200 mmol, except for Entry 9 and 11), $\mathrm{n}(\mathrm{HCOOK}): \mathrm{n}\left(\mathrm{H}_{2} \mathrm{O}\right)=1: 2$, catalyst (Raney nickel, $0.5 \mathrm{~g}$ ), temperature $\left(70{ }^{\circ} \mathrm{C}\right) .{ }^{\mathrm{b}} \mathrm{The}$ amount of HCOOK added is $600 \mathrm{mmol}$. ${ }^{\circ}$ The amount of HCOOK added is $400 \mathrm{mmol}{ }^{\mathrm{d}}$ The product is benzonitrile.

Table 5. Reusability of $\left[\mathrm{DBUH}^{+}\right]\left[\mathrm{C}_{4} \mathrm{H}_{9} \mathrm{COO}^{-}\right]$for transfer hydrogenation of benzonitrile ${ }^{\mathrm{a}}$

\begin{tabular}{lll}
\hline Cycle & Time & Yield $^{\mathrm{b}}(\%)$ \\
\hline 1 & 8 & 89 \\
2 & 8 & 87 \\
3 & 8 & 88 \\
4 & 8 & 87 \\
5 & 8 & 85 \\
6 & 8 & 87 \\
\hline
\end{tabular}

${ }^{\text {a} R e a c t i o n ~ c o n d i t i o n s: ~ I L ~}(50 \mathrm{~mL})$, benzonitrile $(50 \mathrm{mmol})$, HCOOK $(200 \mathrm{mmol})$, water $(400 \mathrm{mmol})$, catalyst (Raney nickel, $0.5 \mathrm{~g})$, temperature $\left(70{ }^{\circ} \mathrm{C}\right) .{ }^{\mathrm{b}} \mathrm{GC}$ yield. 


\section{Conclusions}

Raney nickel catalyzed transfer hydrogenation of nitrile using formate as hydrogen donor was proposed. Compared to previously used hydrogen donors, such as hydrazine and hydrazinium monoformate, formate is less expensive and less hazardous. Three ILs, including $\left[\mathrm{DBUH}^{+}\right]\left[\mathrm{C}_{2} \mathrm{H}_{5} \mathrm{COO}^{-}\right]$, [DBUH $\left.{ }^{+}\right]\left[\mathrm{C}_{3} \mathrm{H}_{7} \mathrm{COO}^{-}\right]$and $\left[\mathrm{DBUH}^{+}\right]\left[\mathrm{C}_{4} \mathrm{H}_{9} \mathrm{COO}^{-}\right]$, were prepared as reaction medium. DBU based IL could promote the transfer hydrogenation obviously, and $\left[\mathrm{DBUH}^{+}\right]\left[\mathrm{C}_{4} \mathrm{H}_{9} \mathrm{COO}^{-}\right]$gave the best performance. Compared with commonly used organic and inorganic solvents, DBU based ILs showed obvious advantage. The preparation of a DBU based IL is simple and the IL can be reused for 6 times at least. The reaction proposed is clean and environmentally benign.

\section{Experimental Section}

\section{General procedure for preparation of ILs $\left[\mathrm{DBUH}^{+}\right]\left[\mathrm{C}_{2} \mathrm{H}_{5} \mathrm{COO}^{-}\right],\left[\mathrm{DBUH}^{+}\right]\left[\mathrm{C}_{3} \mathrm{H}_{7} \mathrm{COO}^{-}\right]$ and $\left[\mathrm{DBUH}^{+}\right]\left[\mathrm{C}_{4} \mathrm{H}_{9} \mathrm{COO}^{-}\right]$}

To a $100 \mathrm{~mL}$ three-necked flask cooled by ice bath, added DBU. Then carboxylic acid (0.9 equiv.) was added dropwise under stirring. After addition, the reaction mixture was stirred at room temperature for $10 \mathrm{~h}$. The unconverted residue was washed by ethyl ether, and the IL was dried under vacuum at $70{ }^{\circ} \mathrm{C}$ for $10 \mathrm{~h}$ to afford a light yellow viscous liquid.

$\left[\right.$ DBUH $\left.^{+}\right]\left[\mathbf{C}_{2} \mathbf{H}_{5} \mathbf{C O O}^{-}\right] .{ }^{1} \mathrm{H}$ NMR $\left(\mathrm{CH}_{3} \mathrm{OH}-d_{4}, 400 \mathrm{MHz}, \mathrm{ppm}\right): \delta=1.08(\mathrm{t}, 3 \mathrm{H}), 1.64-1.84(\mathrm{~m}$, $6 \mathrm{H}), 2.00-2.06(\mathrm{~m}, 2 \mathrm{H}), 2.13(\mathrm{~m}, 2 \mathrm{H}, J=7.6 \mathrm{~Hz}), 2.70-2.81(\mathrm{~m}, 2 \mathrm{H}), 3.35(\mathrm{t}, 2 \mathrm{H}, J=5.7 \mathrm{~Hz})$, $3.45-3.48(\mathrm{~m}, 1 \mathrm{H}), 3.55(\mathrm{t}, 2 \mathrm{H}, J=5.9 \mathrm{~Hz}), 3.60-3.62(\mathrm{~m}, 2 \mathrm{H}) ;{ }^{13} \mathrm{C} \mathrm{NMR}\left(\mathrm{CH}_{3} \mathrm{OH}-d_{4}, 100 \mathrm{MHz}\right.$, ppm): $\delta=10.1,19.1,23.6,26.2,28.6,30.7,32.1,37.9,48.2,53.9,166.0,177.6$; Anal. Calcd for $[\mathrm{DBUH}+]\left[\mathrm{C}_{2} \mathrm{H}_{5} \mathrm{COO}^{-}\right]$: $\mathrm{C} 63.68, \mathrm{H} 9.80, \mathrm{~N} 12.38, \mathrm{O}$ 14.14. Found: $\mathrm{C} 63.71, \mathrm{H} 9.75, \mathrm{~N} 12.42, \mathrm{O}$ 14.03 .

$\left[\mathrm{DBUH}^{+}\right]\left[\mathrm{C}_{3} \mathrm{H}_{7} \mathrm{COO}^{-}\right] .{ }^{1} \mathrm{H}$ NMR $\left(\mathrm{CH}_{3} \mathrm{OH}-d_{4}, 400 \mathrm{MHz}, \mathrm{ppm}\right): \delta=0.93(\mathrm{t}, 3 \mathrm{H}), 1.55-1.65(\mathrm{~m}, 2 \mathrm{H}$, $J=7.4 \mathrm{~Hz}), 1.71-1.83(\mathrm{~m}, 6 \mathrm{H}), 2.00-2.06(\mathrm{~m}, 2 \mathrm{H}), 2.10-2.13(\mathrm{~m}, 2 \mathrm{H}), 2.70-2.80(\mathrm{~m}, 2 \mathrm{H}), 3.35(\mathrm{t}$, $2 \mathrm{H}, J=5.8 \mathrm{~Hz}), 3.44-3.48(\mathrm{~m}, 1 \mathrm{H}), 3.55(\mathrm{t}, 2 \mathrm{H}, J=5.9 \mathrm{~Hz}), 3.59-3.62(\mathrm{~m}, 2 \mathrm{H}) ;{ }^{13} \mathrm{C} \mathrm{NMR}$ $\left(\mathrm{CH}_{3} \mathrm{OH}-d_{4}, 100 \mathrm{MHz}, \mathrm{ppm}\right): \delta=13.3,19.1,19.6,23.6,26.2,28.6,32.1,37.9,40.0,48.2,53.9$, 166.0, 177.6; Anal. Calcd for [DBUH+] $\left[\mathrm{C}_{3} \mathrm{H}_{7} \mathrm{COO}^{-}\right]$: $\mathrm{C}$ 64.97, $\mathrm{H}$ 10.07, N 11.66, O 13.31. Found: C 65.01, H 9.97, N 11.75, O 13.27.

$\left[\right.$ DBUH $\left.^{+}\right]\left[\mathbf{C}_{4} \mathbf{H}_{9} \mathbf{C O O}^{-}\right] .{ }^{1} \mathrm{H}$ NMR $\left(\mathrm{CH}_{3} \mathrm{OH}-d_{4}, 400 \mathrm{MHz}, \mathrm{ppm}\right): \delta=0.91(\mathrm{t}, 3 \mathrm{H}), 1.29-1.39(\mathrm{~m}, 2 \mathrm{H}$, $J=7.3 \mathrm{~Hz}), 1.53-1.60(\mathrm{~m}, 2 \mathrm{H}), 1.65-1.82(\mathrm{~m}, 6 \mathrm{H}), 2.00-2.06(\mathrm{~m}, 2 \mathrm{H}), 2.12-2.16(\mathrm{~m}, 2 \mathrm{H})$, 2.68-2.77 (m, 2H), $3.34(\mathrm{t}, 2 \mathrm{H}, J=5.7 \mathrm{~Hz}), 3.44-3.47(\mathrm{~m}, 1 \mathrm{H}), 3.54(\mathrm{t}, 2 \mathrm{H}, J=5.9 \mathrm{~Hz})$, 3.60-3.61 (m, 2H); ${ }^{13} \mathrm{C} \mathrm{NMR}\left(\mathrm{CH}_{3} \mathrm{OH}-d_{4}, 100 \mathrm{MHz}, \mathrm{ppm}\right): \delta=13.0,19.0,22.5,23.6,26.1,28.58$, 28.6, 32.2, 37.7, 47.8, 53.9, 166.0, 177.6; Anal. Calcd for [DBUH+][C $\left.{ }_{4} \mathrm{H}_{9} \mathrm{COO}^{-}\right]$: $\mathrm{C}$ 66.10, $\mathrm{H}$ 10.30, N 11.01, O 12.58. Found: C 66.21, H 10.24, N 11.10, O 12.45. 


\section{General procedure for catalytic transfer hydrogenation of nitriles}

To a $100 \mathrm{~mL}$ three-necked flask added $\left[\mathrm{DBUH}^{+}\right]\left[\mathrm{C}_{4} \mathrm{H}_{9} \mathrm{COO}^{-}\right](50 \mathrm{~mL})$, nitrile $(50 \mathrm{mmol})$, sodium formate $(250 \mathrm{mmol})$ and Raney nickel $(0.5 \mathrm{~g})$. Then the mixture was heated to $70{ }^{\circ} \mathrm{C}$ and reacted for 8 hours under stirring. After the reaction was completed, the mixture was cooled to $20{ }^{\circ} \mathrm{C}$ and filtered, the filtrate was extracted with ethyl acetate for 3 times. The organic solution was washed by dilute hydrochloric acid, the inorganic layer was separated and neutralized with sodium hydroxide. The inorganic layer was extracted with ethyl acetate, then the product can be obtained after removing ethyl acetate under vacuum at $60{ }^{\circ} \mathrm{C}$. The IL after filtration and extraction was washed by ethyl ether and dried under vacuum at $70^{\circ} \mathrm{C}$ for 2 hours, then it was ready for reuse. The catalyst was recycled by washing the deposit with water and filtering, but it cannot be reused unless it is activated by hydrogen.

Propylamine (1a). Analyzed by GC-MS, MS (EI): m/z (rel. int.) 59 (9), 41 (5), 30 (100), 28 (8), identical with commercial sample.

Butylamine (1b). Analyzed by GC-MS, MS (EI): m/z (rel. int.) 73 (7), 30 (100), 28 (5), identical with commercial sample.

Benzylamine (1c). ${ }^{1} \mathrm{H}$ NMR $\left(\mathrm{CDCl}_{3}, 400 \mathrm{MHz}, \mathrm{ppm}\right): \delta=1.34$ (s, $\left.2 \mathrm{H}\right), 3.62(\mathrm{~s}, 2 \mathrm{H}), 7.07-7.19$ (m, 5H); ${ }^{13} \mathrm{C} \mathrm{NMR}\left(\mathrm{CDCl}_{3}, 100 \mathrm{MHz}, \mathrm{ppm}\right): \delta=46.2,126.5,126.9,128.3,143.4{ }^{15}$.

4-Methylbenzylamine (1d): ${ }^{1} \mathrm{H} \mathrm{NMR}\left(\mathrm{CDCl}_{3}, 400 \mathrm{MHz}, \mathrm{ppm}\right): \delta=1.45$ (brs, $\left.2 \mathrm{H}\right), 2.32$ (s, 3H), $3.89(\mathrm{~s}, 2 \mathrm{H}), 7.12(\mathrm{~d}, 2 \mathrm{H}, J=8.0 \mathrm{~Hz}), 7.20(\mathrm{~d}, 2 \mathrm{H}, J=8.0 \mathrm{~Hz}) ;{ }^{13} \mathrm{C} \mathrm{NMR}\left(\mathrm{CDCl}_{3}, 100 \mathrm{MHz}\right.$, ppm): $\delta=21.2,46.0,126.8,129.7,136.0,140.7^{16}$.

2-Methylbenzylamine (1e). ${ }^{1} \mathrm{H} \mathrm{NMR}\left(\mathrm{CDCl}_{3}, 400 \mathrm{MHz}, \mathrm{ppm}\right): \delta=1.64$ (brs, $\left.2 \mathrm{H}\right), 2.31$ (s, 3H), $3.78(\mathrm{~s}, 2 \mathrm{H}), 7.15-7.22(\mathrm{~m}, 3 \mathrm{H}), 7.27(\mathrm{~d}, 1 \mathrm{H}, J=6.7 \mathrm{~Hz}) ;{ }^{13} \mathrm{C} \mathrm{NMR}\left(\mathrm{CDCl}_{3}, 100 \mathrm{MHz}, \mathrm{ppm}\right)$ : $\delta=18.7,43.9,126.1,126.7,126.9,130.2,135.4,141.1^{17}$.

4-Methoxybenzylamine (1g). ${ }^{1} \mathrm{H}$ NMR $\left(\mathrm{CDCl}_{3}, 400 \mathrm{MHz}, \mathrm{ppm}\right): \delta=1.52$ (brs, $\left.2 \mathrm{H}\right), 3.73$ (s, $3 \mathrm{H}), 3.76(\mathrm{~s}, 2 \mathrm{H}), 6.84(\mathrm{~d}, 2 \mathrm{H}, J=8.8 \mathrm{~Hz}), 7.17(\mathrm{~d}, 2 \mathrm{H}, J=8.5 \mathrm{~Hz}) ;{ }^{13} \mathrm{C} \mathrm{NMR}\left(\mathrm{CDCl}_{3}, 100 \mathrm{MHz}\right.$, ppm): $\delta=45.7,55.2,114.0,128.5,134.1,158.7^{15}$.

2-Phenethylamine (1h). ${ }^{1} \mathrm{H}$ NMR $\left(\mathrm{CDCl}_{3}, 400 \mathrm{MHz}, \mathrm{ppm}\right): \delta=1.19$ (s, 2H), 2.75 (t, 2H), 2,89 (t, $2 \mathrm{H})$, 7.02-7.37 (m, $5 \mathrm{H}){ }^{18} ;{ }^{13} \mathrm{C} \mathrm{NMR}\left(\mathrm{CDCl}_{3}, 100 \mathrm{MHz}, \mathrm{ppm}\right): \delta=40.5,44.2,126.1,128.0,128.7$, $140.2^{19}$.

4-aminobenzylamine (1i). ${ }^{1} \mathrm{H}$ NMR $\left(\mathrm{CDCl}_{3}, 400 \mathrm{MHz}, \mathrm{ppm}\right): \delta=2.02(\mathrm{brs}, 2 \mathrm{H}), 3.61(\mathrm{~s}, 2 \mathrm{H})$,

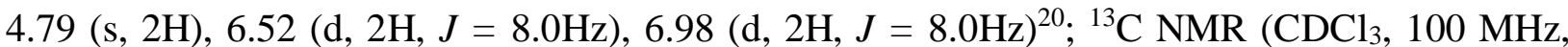
ppm): $\delta=46.1,115.0,128.4,132.9,147.2^{21}$.

(1H-indol-2-yl)methanamine (1j). ${ }^{1} \mathrm{H}$ NMR (DMSO- $d_{6}, 400 \mathrm{MHz}, \mathrm{ppm}$ ): $\delta=3.84$ (s, 2H), 6.22 $(\mathrm{s}, 1 \mathrm{H}), 6.93(\mathrm{t}, 1 \mathrm{H}, J=8.0 \mathrm{~Hz}), 7.01(\mathrm{t}, 1 \mathrm{H}, J=7.8 \mathrm{~Hz}), 7.30(\mathrm{~d}, 1 \mathrm{H}, J=7.8 \mathrm{~Hz}), 7.44(\mathrm{~d}, 1 \mathrm{H}, J$ $=8.0 \mathrm{~Hz}$ ), $8.57(\mathrm{~s}, 2 \mathrm{H}), 10.94$ (brs, $1 \mathrm{H}) ;{ }^{13} \mathrm{C}$ NMR (DMSO- $\left.d_{6}, 100 \mathrm{MHz}, \mathrm{ppm}\right): \delta=57.4,98.2$, $111.1,118.9,119.6,120.4,128.5,136.4,141.8^{22}$. 
1,3-Phenylenedimethanamine (1k). ${ }^{1} \mathrm{H}$ NMR (DMSO- $d_{6}, 400 \mathrm{MHz}, \mathrm{ppm}$ ): $\delta=2.59$ (brs, $4 \mathrm{H}$ ), $3.73(\mathrm{~s}, 4 \mathrm{H}), 7.18(\mathrm{~d}, 2 \mathrm{H}, J=7.8 \mathrm{~Hz}), 7.24(\mathrm{dd}, 1 \mathrm{H}, J=6.7,6.5 \mathrm{~Hz}), 7.31(\mathrm{~s}, 1 \mathrm{H}) ;{ }^{13} \mathrm{C} \mathrm{NMR}$ (DMSO- $d_{6}, 100 \mathrm{MHz}, \mathrm{ppm}$ ): $\delta=46.1,125.3,126.1,128.3,144.2$. EI-HRMS calcd for $\mathrm{C}_{8} \mathrm{H}_{12} \mathrm{~N}_{2}$ $\left(\mathrm{M}^{+}\right)$: 136.1000, found: 136.1002 .

\section{Acknowledgements}

We acknowledge the funding support by a grant from the National Natural Science Foundation of China $(21076183,21006087)$, and we are also grateful to Dr. Ying, A.G., who have given us important suggestions and provided us materials for ionic liquid preparation.

\section{References}

1. (a) Addis, D.; Enthaler, S.; Junge, K.; Wendt, B.; Beller, M. Tetrahedron Lett. 2009, 50, 3654. (b) Enthaler, S.; Junge, K.; Addis, D.; Erre, G.; Beller, M. Chem. Sus. Chem. 2008, 1(12), 1006. (c) Enthaler, S.; Junge, K.; Addis, D.; Erre, G.; Beller, M. Chem. Eur. J. 2008, $14,9191$.

2. Xie, X.; Liotta, C. L.; Eckert, C. A. Ind. Eng. Chem. Res. 2004, 43, 7907.

3. Sarmah, B. K.; Barua, N. C., Tetrahedron 1991, 47, 8587.

4. Suda, K.; Hino, F.; Yijima, C., J. Org. Chem. 1986, 51, 4232.

5. Mebane, R. C.; Jensen, D. R.; Rickerd, K. R.; Gross, B. H. Synthetic. Commun. 2003, 33, 3373.

6. Mizushima, E.; Yamaguchi, M.; Yamagishi, T. J. Mol. Catal. a-Chem. 1999, 148 (1-2), 69.

7. Moiseev, I. I.; Tsirkov, G. A.; Gekhman, A. E.; Vargaftik, M. N. Mendeleev. Commun. 1997, (1), 1.

8. Zajac, W. W.; Siuda, J. F.; Nolan, M. J.; Santosus.Tm. J. Org. Chem. 1971, 36, 3539.

9. You, Q. D.; Zhou, H. Y.; Wang, Q. Z.; Lei, X. H. Acta Chim. Sinica 1993, 51, 85.

10. (a) Gowda, S.; Gowda, D. C. Tetrahedron 2002, 58, 2211. (b) Gowda, S.; Gowda, B. K. K.; Gowda, D. C. Synthetic. Commun. 2003, 33, 281.

11. (a) Hut'ka, M.; Toma, S. Monatsh. Chem. 2008, 139, 793. (b) Hut'ka, M.; Toma, S. Monatsh. Chem. 2009, 140, 1189. (c) Baan, Z.; Finta, Z.; Keglevich, G.; Hermecz, I. Green Chem. 2009, 11, 1937. (d) Kawasaki, I.; Tsunoda, K.; Tsuji, T.; Yamaguchi, T.; Shibuta, H.; Uchida, N.; Yamashita, M.; Ohta, S. Chem. Commun. 2005, 2134.

12. Ying, A. G.; Wang, L. M.; Deng, H. X.; Chen, J. H.; Chen, X. Z.; Ye, W. D. Arkivoc 2009, (xi), 288.

13. Ying, A. G.; Liu, L.; Wu, G. F.; Chen, X. Z.; Ye, W. D.; Chen, J. H.; Zhang, K. Y. Chem. Res. Chinese U. 2009, 25, 876. 
14. Vanes, T.; Staskun, B. Org. Synth. 1988, 50-9, 631.

15. Haddenham, D.; Pasumansky, L.; DeSoto, J.; Eagon, S.; Singaram, B. J. Org. Chem. 2009, 74, 1964.

16. Kruger, T.; Vorndran, K.; Linker, T. Chem-Eur. J. 2009, 15, 12082.

17. Huang, W. H.; Zavalij, P. Y.; Isaacs, L. Org. Lett. 2008, 10, 2577.

18. Cho, S. D.; Song, S. Y.; Kim, K. H.; Zhao, B. X.; Ahn, C.; Joo, W. H.; Yoon, Y. J.; Falck, J. R.; Shin, D. S. B Kor. Chem. Soc. 2004, 25, 415.

19. Border, C. L.; Craik, D. J.; Shehan, B. P. Magn. Reson. Chem. 1993, 31, 222.

20. King, J. F.; Rathore, R.; Lam, J. Y. L.; Guo, Z. R.; Klassen, D. F. J. Am. Chem. Soc. 1992, $114,3028$.

21. Brown, N. C.; Gambino, J.; Wright, G. E. J. Med. Chem. 1977, 20, 1186.

22. Fodor, L.; Csomos, P.; Mandity, I.; Bernath, G. Tetrahedron 2007, 63, 4983. 